\title{
Nicotinic Acetylcholine Receptor-like Molecules in the Retina, Retinotectal Pathway, and Optic Tectum of the Frog
}

\author{
Peter B. Sargent, ${ }^{1,2}$ Susan H. Pike, ${ }^{2 . a}$ Deborah B. Nadel, ${ }^{2, b}$ and Jon M. Lindstrom ${ }^{3}$ \\ 'Division of Biomedical Sciences, University of California, Riverside, California 92521, '2Department of Cell Biology, \\ Stanford University School of Medicine, Stanford, California 94305, and ${ }^{3}$ The Salk Institute for Biological Studies, San \\ Diego, California 92138
}

\begin{abstract}
Forty-two monoclonal antibodies (mAbs) generated against nicotinic acetylcholine receptors (AChRs) from electric organ were tested for their ability to cross-react in the optic tectum of the frog Rana pipiens. Twenty-eight of the mAbs tested $(67 \%)$ bound to the optic neuropil of the tectum as revealed by immunoperoxidase cytochemistry. The pattern of peroxidase stain for cross-reacting mAbs corresponded in position to a subset of the retinotectal projections. Electron microscopic examination revealed that peroxidase reaction product was associated with the surface of vesiclecontaining profiles but not with synaptic sites. Removal of one retina resulted in the loss of immunoreactivity in the contralateral tectum. AChR-like immunoreactivity was also associated with the optic tract and optic nerve and with retinal ganglion cells.

These results indicate that some classes of retinal ganglion cells bear AChR-like molecules on their surface. The existence of these molecules on ganglion cell axons and terminals seems the most likely explanation for the AChRlike immunoreactivity present in the tectum.
\end{abstract}

Nicotinic acetylcholine receptors (AChRs) from skeletal muscle and nervous tissue belong to the same gene family and share homologies in subunit cDNA sequence as well as epitopes recognized by common monoclonal antibodies (mAbs) (reviewed by Lindstrom et al., 1987). However, AChRs from muscle and nerve differ in pharmacological properties, subunit structure, and, possibly, functional role. Obvious pharmacological differences include the fact that muscle AChRs bind $\alpha$-bungarotoxin and have relatively low affinity for nicotine, whereas many neuronal AChRs do not bind $\alpha$-bungarotoxin and have very high

\footnotetext{
Received Mar. 3, 1988; revised June 13, 1988; accepted June 17, 1988.

This work was supported by NSF Grant BNS 83-05694 and NIH Grants NS 22491 and NS 24207 (to P.B.S.), and by NIH Grant NS 11323 , the Muscular Dystrophy Association, the Alexander Onassis Public Benefit Foundation, the U.S. Army (DAMD17-86-C-6148), the Los Angeles and California Chapters of the Mysathenia Gravis Foundation, and the Council for Tobacco Research (to J.M.L.). We thank Larisa Tsavaler for her participation in some of the early experiments and Drs. Larry Swanson and Thom Hughes for reviewing a preliminary version of the manuscript. We also wish to thank Dr. Robert Sealock (University of North Carolina) for Naja naja siamensis toxin and rabbit anti-N.n.s. toxin and Dr. Jack McMahan (Stanford University) for HRP- $\alpha$-bungarotoxin.

Correspondence should be addressed to Dr. Peter B. Sargent, Division of Biomedical Sciences, University of California, Riverside, CA 92521.

a Present address: Institute of Neuroscience, University of Oregon, Eugene, OR 97403.

' Present address: School of Medicine, University of California, San Fransisco, CA 94143

Copyright (C) 1989 Society for Neuroscience $0270-6474 / 89 / 020565-09 \$ 02.00 / 0$
}

affinity for nicotine. Muscle AChRs are composed of 4 kinds of subunits, whereas neuronal nicotinic AChRs that do not bind $\alpha$-bungarotoxin are composed of only 2 kinds. AChRs in the postsynaptic membrane of muscle are a critical link in neuromuscular transmission, and while some neuronal nicotinic AChRs, such as those in the ciliary ganglion, have a similar postsynaptic role, much evidence suggests that many neuronal nicotinic AChRs in brain are located presynaptically and may modulate release of other transmitters (reviewed by Lindstrom et al., 1987). This study uses mAbs that cross-react between AChRs of muscle and nerve to demonstrate that AChR-like molecules are present on frog retinal ganglion cells and their central projections, as has been previously demonstrated in fish (Henley et al., 1986a, b), chickens (Swanson et al., 1987; Keyser et al., 1988), and rats (Swanson et al., 1987). This study extends AChR localization to the ultrastructual level and reveals that AChRs within the optic tectum are extrasynaptic, as would be expected if they were located on the terminals of retinal afferents.

A preliminary account of these results has appeared (Sargent et al., 1984b).

\section{Materials and Methods}

Animals. Most of the experiments were done on Rana pipiens (body length of $5.0-7.5 \mathrm{~cm}$, either sex) obtained from Hazen Co. (Alburg, VT). Common goldfish (Crassius auratus, $4.0-6.0 \mathrm{~cm}$ total length) were obtained from a local supplier.

Reagents. Monoclonal antibodies against electric organ AChR were made in rats and characterized as described in Tzartos and Lindstrom (1980) and Tzartos et al. (1981, 1986). Biotinylated rabbit anti-rat immunoglobulin (IgG) and avidin-biotinylated HRP complex were purchased in kit form (Vectastain) from Vector Laboratories. Naja naja siamensis toxin (cobratoxin) and rabbit anti-cobratoxin were kindly provided by Dr. Robert Sealock, University of North Carolina. ${ }^{125} I-\alpha-$ bungarotoxin was prepared and purified by the technique of Lindstrom et al. (1981). Fluorescein-goat-anti-rat IgG was obtained from Cappel Laboratories (Organon Teknika). All other reagents were purchased from Sigma Chemical Company unless indicated otherwise.

Surgery. All surgical procedures were performed following anesthesia by immcrsion of animals in $2 \mathrm{~mm}$ tricaine methanesulfonate. The optic nerve was cut after approaching it through the soft palate. The soft palate was then sutured with 6-0 monofilament nylon thread. Retinas were eviscerated by aspiration following removal of the lens and vitreous body (Matsumoto and Scalia, 1981). The eyelid was then sutured shut. The projection of retinal ganglion cells to the tectum was labeled by placing crystals of HRP (Sigma, type VI) in contact with the central stump of the severed optic nerve and examining the tectum 2-3 d later.

Immunocytochemistry: general procedures. Light microscopic immunocytochemistry was performed on frozen or Vibratome sections of tecta of animals fixed by perfusion with periodate-lysine-paraformaldehyde (PI P) containing 2\% paraformaldehyde (McLean and Nakane, 1974 ) or with $4 \%$ acrolein in $90 \mathrm{~mm}$ Na phosphate buffer (King et al., 1983). The avidin biotinylated-peroxidase complex (ABC) method of 
Hsu et al. (1981) was used to visualize primary antibody binding, and peroxidase was revealed colorimetrically by the cobalt-glucose oxidase proccdurc of Itoh ct al. (1979).

Animals were perfused through the truncus arteriosus first with frog Ringer's containing $2 \mathrm{~mm}$ tricaine methanesulfonate until fluid returning to the heart was clear and subsequently with fixative for 7.5 (acrolein) or 90 (PLP) min. The brain with optic nerves was then removed and immersion-fixed for an additional 7.5 (acrolein) or 30 (PLP) min. For frozen sections the brain was then equilibrated in $30 \%$ sucrose in 90 $\mathrm{mm} \mathrm{Na}$ phosphate buffer, frozen by immersion in liquid nitrogen, and mounted in Tissue Tek mounting medium (Miles Laboratories) prior to sectioning at $10 \mu \mathrm{m}$ in a Hacker/Bright microtome cryostat. Sections were placed on subbed glass slides and air-dried for $30 \mathrm{~min}$ before use. For Vibratome sections, the brain was embedded in $4 \%$ agar and sectioned at $100 \mu \mathrm{m}$ using a Lancer series 100 Vibratome. In a separate set of experiments, retinas were Vibratome-sectioned after being removed from the sclera of an anesthetized frog and immersion-fixed in PLP for $30 \mathrm{~min}$.

Immunocytochemistry for light microscopy. Vibratome sections of tissue were processed "free-floating" according to following schedule (all steps were done at room temperature unless indicated otherwise). Frozen sections were processed mounted and for shorter times ( $1 \mathrm{hr}$ for antibody incubations, $30 \mathrm{~min}$ for washes).

1. Pre-incubate Vibratome sections in frog Ringer's containing 3\% normal rabbit serum and $0.025 \%$ saponin (RRS) for $30 \mathrm{~min}$.

2. Incubate sections in primary antibody diluted to a titer of 10-200 nM in RRS for $16 \mathrm{hr}$ at $4^{\circ} \mathrm{C}$.

3. Wash in RRS for $2-3 \mathrm{hr}$, changing the solution every $20 \mathrm{~min}$.

4. Incubate the sections in biotinylated rabbit anti-rat IgG diluted $1: 200$ in RRS for $6 \mathrm{hr}$.

5. Wash in RRS for $2-3 \mathrm{hr}$, changing the solution cvery $20 \mathrm{~min}$.

6 . Incubate sections in avidin-HRP, diluted 1:100 in RRS, for $16 \mathrm{hr}$ at $4^{\circ} \mathrm{C}$.

7. Wash in RRS for $1 \mathrm{hr}$, changing the solution every $20 \mathrm{~min}$.

8 . Wash in Ringer's for $1 \mathrm{hr}$, changing the solution every $20 \mathrm{~min}$.

9. Fix the sections in $1 \%$ glutaraldehyde in $60 \mathrm{~mm}$ sodium phosphate buffer, $\mathrm{pH} 7.2$, for $1 \mathrm{hr}$.

10. Rinse in Ringer's, $15 \mathrm{~min}$.

11. Preincubate the sections in a solution containing diaminobenzidine, nickel ammonium sulfate, cobalt chloride, and $\beta$-D-glucose in phosphate buffer (final concentrations: diaminobenzidine, $0.5 \mathrm{mg} / \mathrm{ml}$; nickel ammonium sulfate, $0.025 \%$; cobalt chloride, $0.025 \%, \beta$-D-glucose, $2 \mathrm{mg} / \mathrm{ml}$ ) for $15 \mathrm{~min}$.

12. Incubate the sections in a solution identical to the preincubation solutions but containing, in addition, $0.2 \mathrm{mg} / \mathrm{ml}$ ammonium chloride and $0.003 \mathrm{mg} / \mathrm{ml}$ glucose oxidase (Sigma, Type II) until there is adequate staining intensity.

13. Rinse the sections in Ringer's for $15 \mathrm{~min}$.

14. Mount the sections in $90 \%$ glycerol $/ 10 \%$ Ringer's.

In a few instances immunoperoxidase experiments were performed on only one side of the tectum in order to compare the pattern of staining with the pattern of the retinotectal projection (the retinotectal projection is almost completely crossed). HRP crystals were placed in contact with the central cut end of the left optic nerve in an anesthetized frog. Two to three days later the animal was reanesthetized and perfused with fixative. The tectum was then serially sectioned, with the plane of the sections being as close as possible to coronal. Each section was then split in the midsagittal plane, and each right half-section was set aside. The left half-scctions wcrc reacted for AChR-likc immunoreactivity (through step 10, above), and both right and left halves were reacted for HRP. The matching sections were then reassembled photographically, thus producing a reconstituted tectal slice in which the retinal projection appeared on the right half and the AChR-like immunoreactivity appeared on the left.

Immunocytochemistry for electron microscopy. The procedure for performing immunocytochemical staining for analysis in the electron microscope was somewhat similar to that for light microscópy. Only Vibratome sections were used, and saponin was omitted in order to preserve adequate fine structure. Electron microscopic analysis was performed after cutting thin sections orthogonal to the plane of the Vibratome section. The heaviest peroxidase stain was found at the surface of the Vibratome section, but the tissue was poorly preserved. The best results were obtained $1-3 \mu \mathrm{m}$ into the section, where peroxidase staining was still strong and where tissue preservation was adequate.

Electron microscopic analysis was performed on sections treated as above (steps 1-13) and postfixed in $1 \% \mathrm{OsO}_{4}$ in $90 \mathrm{~mm}$ sodium phosphate buffer, $\mathrm{pH} 7.2$, dehydrated, and embedded in Epon/Araldite. Sections having a silver intcrferencc color $(70-80 \mathrm{~nm})$ were cut on a Reichert Ultracut ultramicrotome and examined without grid staining on a Philips $200 \mathrm{EM}$. Contrast was sometimes enhanced by adding tannic acid to the glutaraldehyde step (no. 9 , above) to a final concentration of $0.3 \%$ (Wray and Sealock, 1984).

In a few experiments Vibratome sections of tectum were incubated in concentrated solutions of the tracers ferritin or $6 \mathrm{~nm}$ colloidal goldprotein A. Cationized ferritin (Polysciences) was used at $20 \mathrm{mg} / \mathrm{ml}$ in Ringer's. Colloidal gold-protein A was prepared according to the technique of Mühlpfordt (1982), conjugated to protein A (Pharmacia P-L Biochemicals), purified after Slot and Geuze (1981) and used at an O.D. (a) $525 \mathrm{~nm}$ of 1.0 in Ringer's. Tissue incubated for $1 \mathrm{hr}$ with tracer was fixed in $1 \%$ glutaraldehyde in $60 \mathrm{~mm} \mathrm{Na}$ phosphate without tracer washout. The purpose of these experiments was to learn the extent to which these rcagents penctrated the Vibratome slice and the cell profiles within its interior.

Immunoprecipitation experiments. In a few experiments we sought to learn whether anti-AChR antibodies might immunoprecipitate ${ }^{125} \mathbf{I}-$ $\alpha$-bungarotoxin binding sites from extracts of tectal homogenates (see Henley et al., 1986a). Midbrains from either frog or goldfish were homogenized in $50 \mathrm{~mm}$ Tris aminomethanesulfonate, $\mathrm{pH} 7.2,5 \mathrm{~mm}$ iodoacetamide and $1 \mathrm{~mm}$ phenylmethylsulfonyl fluoride (hereafter, HB) and spun for $5 \mathrm{~min}$ in a Beckman Type 65 rotor at $5200 \mathrm{rpm}$. The supernatant was then pelleted by centrifugation in the Type 65 rotor for $1 \mathrm{hr}$ at 25,000 rpm. The pellet was taken up in HB containing $1 \%$ Triton X-100 and gently shaken for $30 \mathrm{~min}$ at $4^{\circ} \mathrm{C}$, and the extract was clarified by centrifugation for $1 \mathrm{hr}$ (as above). The extract was then assayed by incubating fractions with $5 \mathrm{nM}{ }^{125} \mathrm{I}-\alpha$-bungarotoxin and simultancously with mAb no. 22 or 32 (final titer, $100 \mathrm{~nm}$ ) overnight at $4^{\circ} \mathrm{C}$ and then by immunoprecipitating the fraction with goat anti-rat antiserum. Precipitates were pelleted in a microfuge tube and washed twice before being counted in a Beckman gamma counter. All assays were done in triplicate.

\section{Results}

Two-thirds of $42 \mathrm{mAbs}$ tested produced a detectable immunoperoxidase staining pattern within the optic tectum. Figure $1 A$, depicting a typical result, shows peroxidase stain within the superficial layers of the tectum using mAb 22. Figure $1, B, C$, shows the staining pattern observed at higher magnification in micrographs taken using Nomarski interference contrast optics; the staining produced by mAb 22 (Fig. $1 C$ ) is compared with that produced by $\mathrm{mAb} 32$ (Fig. $1 B$ ). The use of $\mathrm{mAb} 22$, but not $\mathrm{mAb} 32$, produced a strong peroxidase stain in the superficial layers of the tectum. Both antibodies were made against AChR from Electrophorus electricus; mAb 32, however, does not cross-react with AChR from either Torpedo californica electric organ (Tzartos et al., 1981) or Rana pipiens skeletal muscle (Sargent et al., 1984a), whereas mAb 22 cross-reacts with both. $\mathrm{mAb} 32$, which is the same isotype as mAb 22 ( $\operatorname{IgG} 2 b)$, is an ideal control for binding specificity.

Table 1 summarizes the results obtained with the $42 \mathrm{mAbs}$ tested. All of the cross-reacting $m A$ bs produced a similar pattern of staining (Fig. 1), although there was considerable variability among mAbs in staining intensity. All of the mAbs that produced a strong pattern of immunoperoxidase staining are specific for the main immunogenic region (MIR), a highly conserved and highly immui ogenic region of the extracellular surface of the AChR's $\alpha$ subunit. Every one of the 22 anti-MIR mAbs tested that cross-reacted with AChRs in Rana skeletal muscle produced immunoperoxidase staining when used in the tectum, and the reaction product was usually strong (as in Fig. 1). By contrast, only a few (6) of the 16 other mAbs tested produced detectable staining in the tectum, and positive results were generally obtained for each in fewer than half of the trials. 
Table 1. Anti-AChR binding in the optic tectum of Rana pipiens

\begin{tabular}{|c|c|c|c|c|c|}
\hline $\begin{array}{l}\text { mAb } \\
\text { no. }\end{array}$ & $\begin{array}{l}\text { Subunit } \\
\text { specificity }\end{array}$ & $\begin{array}{l}\text { Binding to } \\
\text { AChRs in } \\
\text { Rana } \\
\text { muscle }\end{array}$ & $\begin{array}{l}\text { Binding to } \\
\text { Rana tectum }\end{array}$ & $\begin{array}{l}\text { No. of } \\
\text { Exps. }\end{array}$ & $\begin{array}{l}\text { Positive } \\
\text { experi- } \\
\text { ments } \\
(\%)\end{array}$ \\
\hline 4 & MIR & + & + (weak) & 5 & 40 \\
\hline 6 & $\alpha$, MIR & + & + (strong) & 5 & 80 \\
\hline 7 & $\delta, \gamma$ & + & - & 7 & 0 \\
\hline 8 & $\alpha$ & + & + (weak) & 2 & 50 \\
\hline 11 & $\beta$ & + & - & 5 & 0 \\
\hline 12 & MIR & - & - & 2 & 0 \\
\hline 16 & $\alpha$, MIR & - & - & 2 & 0 \\
\hline 17 & $\alpha$, MIR & + & + (strong) & 7 & 100 \\
\hline 19 & $\alpha$, MIR & - & - & 2 & 0 \\
\hline 21 & $\alpha$, MIR & + & + (weak) & 2 & 50 \\
\hline 22 & $\alpha$, MIR & + & + (strong) & 11 & 100 \\
\hline 24 & $\alpha$, MIR & + & + (weak) & 4 & 75 \\
\hline 28 & $\alpha$, MIR & + & + (strong) & 6 & 100 \\
\hline 31 & $\alpha$, MIR & + & + (strong) & 2 & 100 \\
\hline 32 & $\alpha$, MIR & - & - & 4 & 0 \\
\hline 35 & $\alpha$, MIR & + & + (strong) & 6 & 100 \\
\hline 36 & $\alpha$, MIR & + & + (strong) & 3 & 100 \\
\hline 37 & $\alpha$, MIR & + & + (strong) & 16 & 100 \\
\hline 38 & $\alpha$, MIR & + & + (strong) & 10 & 100 \\
\hline 39 & $\alpha$, MIR & + & + (strong) & 5 & 100 \\
\hline 41 & $\alpha$, MIR & + & + (strong) & 8 & 100 \\
\hline 42 & $\alpha$, MIR & + & + (weak) & 3 & 33 \\
\hline 44 & $\alpha$, MIR & + & + (strong) & 3 & 100 \\
\hline 46 & $\alpha$, MIR & + & + (strong) & 3 & 100 \\
\hline 47 & $\alpha$, MIR & + & + (strong) & 4 & 100 \\
\hline 50 & $\alpha$, MIR & + & + (strong) & 3 & 100 \\
\hline 60 & $\delta$ & + & - & 2 & 0 \\
\hline 61 & $\alpha$ & + & - & 4 & 0 \\
\hline 94 & $\beta$ & + & - & 3 & 0 \\
\hline 110 & $\beta$ & + & + (weak) & 3 & 33 \\
\hline 111 & $\beta$ & + & + (weak) & 8 & 25 \\
\hline 113 & $\beta$ & + & - & 2 & 0 \\
\hline 118 & $\beta$ & + & - & 3 & 0 \\
\hline 123 & $\beta$ & + & - & 3 & 0 \\
\hline 124 & $\beta, \gamma$ & + & - & 2 & 0 \\
\hline 139 & $\delta$ & + & + (weak) & 11 & 18 \\
\hline 142 & $\alpha, \beta, \gamma, \delta$ & + & + (weak) & 7 & 57 \\
\hline 154 & $\gamma$ & + & + (weak) & 10 & 30 \\
\hline 168 & $\gamma, \beta$ & + & - & 2 & 0 \\
\hline 176 & MIR & + & + (strong) & 3 & 100 \\
\hline 177 & MIR & + & + (strong) & 5 & 100 \\
\hline 188 & MIR & + & + (strong) & 4 & 100 \\
\hline
\end{tabular}

All mAbs were made against electric organ $\mathrm{AChR}$ or $\mathrm{AChR}$ subunits and characterized as described in Tzartos and Lindstrom (1980) and Tzartos et al $(1981,1986)$. Reactivity with AChRs in skeletal muscle was determined by Sargent et al. (1984a). Binding in the optic tectum was tested 2-16 times for each mAb. Peroxidase staining with some cross-reacting mAbs was strong and consistently observed, whereas for others it was weak and inconsistently observed.

The frog optic tectum is divided into layers alternating between cell-rich and cell-poor (see Fig. $1 B$ ). Layer 1, which borders the ventricle, consists of ependymal cells. Layers 2, 4, 6, and 8 are enriched in cell bodies, while layers 3, 5, 7, and 9 are enriched in neuropil. All of the detectable AChR-like immunoreactivity within the tectum lies within layer 9 . At low mag-
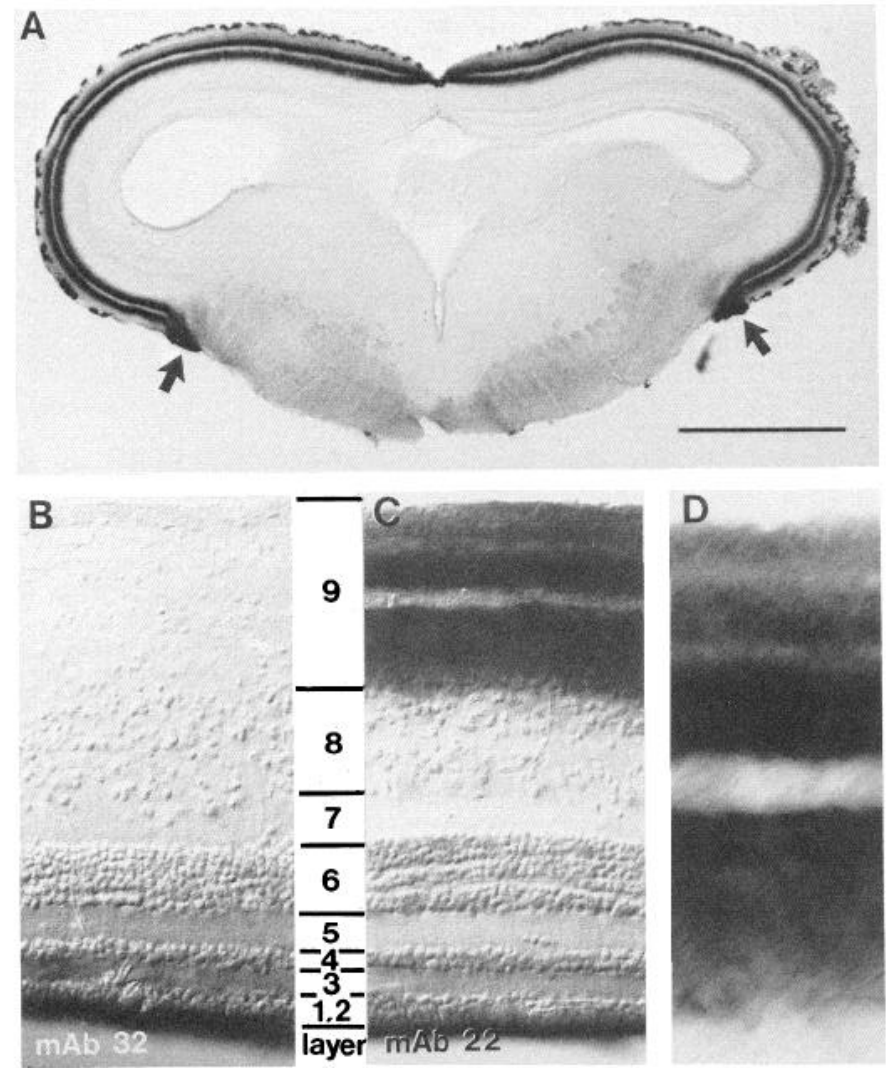

Figure 1. Immunoperoxidase staining of the optic tectum using antiAChR antibody. A, Low-power bright-field photomicrograph of a 100 $\mu \mathrm{m}$ Vibratome section from the midbrain of Rana pipiens. Peroxidase staining is found within the superficial parts of the tectum and extends to the lateral optic tracts (arrows; see also Fig. 2). Density at the base of the tectum (between the 2 arrows) represents cobalt staining of myelin tracts and not HRP reaction product. $B$ and $C$, Higher-power differential interference contrast (Nomarski) photomicrographs after incubating Vibratome sections with $\mathrm{mAb} 32(B)$ and $\mathrm{mAb} 22(C)$ and visualizing $\mathrm{mAb}$ binding using the avidin biotinylated-HRP technique (Hsu et al., 1981). Strong staining is observed after using $\mathrm{mAb} 22$, but not $\mathrm{mAb} 32$ (density at bottom of slices is shadowing due to Nomarski optics). The vertically oriented set of numbers between $B$ and $C$ refer to the layers of the tectum, extending from the ventricular surface (layer 1) to the pia (layer 9). $D$, Banding pattern of stain within the neuropil at high magnification (bright-field optics). The entire field in $D$ corresponds to layer 9. Distinct bands of stain are visible. Scale bar (in $A$ ): $1 \mathrm{~mm}$ in $A, 150 \mu \mathrm{m}$ in $B$ and $C$, and $60 \mu \mathrm{m}$ in $D$.

nification the immunoreactivity is apparent as 2 bands separated by an unstained area (Fig. 1A). At higher magnification, however, it is apparent that the superficial band of stain is divided into layers (Fig. 1D). The retinal projection to the tectum is itself composed of several layers, each of which apparently represents a complete, topologically ordered projection from the eye to the tectum; each projection terminates in a separate layer within the tectum and is characterized by distinct functional properties (Maturana et al., 1960). The anatomically distinct projection bands are named " $a$ " through " $g$ " according to the nomenclature of Potter (1969), with layer "a" closest to the pia. Projection layers "a" through "f" are located in layer 9, and layer "g" at the border of layers 7 and 8 . In order to compare in detail the retinal projection with the immunoperoxidase staining pattern observed with anti-AChR mAbs, we labeled the projection on one side of the tectum and compared it with 

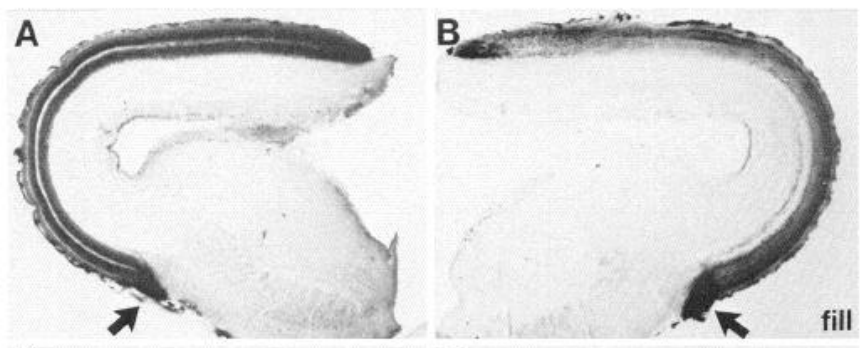

C
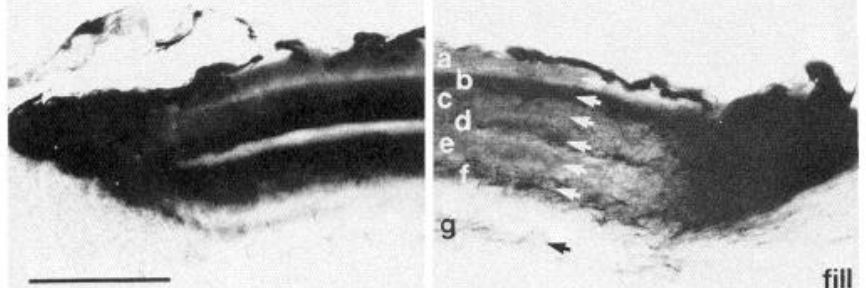

Figure 2. Comparison of immunoperoxidase staining pattern $(A, C)$ with projection of retinal afferents $(B, D)$. $A$, Pattern of AChR immunoreactivity using mAb 22 in a half tectum. $B$, Retinal projection to the contralateral half of the same tectal slice (experimental details given in Materials and Methods). The immunoreactivity and the projection are similar but not identical. The lateral optic tract $(B$, arrow $)$ is stained in $A$ (arrow). $C$ and $D$, Higher-power views of comparable regions of matching half slices like those shown in $A$ and $B$. The immunoperoxidase stain $(C)$ is compared to the projection $(D)$. The projection is divided into 7 distinct layers (" $a-g$ "), 3 of which are unmyelinated (" $a$," " $c$," and " $e$ ") and 4 of which are myelinated. There is a rough correspondence between bands of peroxidase stain and individual layers of the projection. Individual peroxidase-stained bands appear to line up with projection layers " $a-c$," and the broad, deep peroxidase-stained band lines up with projection layers " $e$ " and " $f$." There appears to be no peroxidase stain associated with projection layers " $d$ " and " $g$." Scale bar (in $C$ ): $1 \mathrm{~mm}$ in $A$ and $B ; 150 \mu \mathrm{m}$ in $C$ and $D$.

the peroxidase staining on the contralateral side. A typical result is shown in Figure 2, $A, B$. The projection is shown on the right ("fill") and the immunoperoxidase staining on the left. The patterns are clearly similar. A closer examination of parts of each side which have been photographically truncated and apposed (Fig. 2, $C, D$ ) reveals that the immunoperoxidase stain occupies the same area of neuropil as does layers "a" through " $f$ " of the projection. The 3 most superficial bands of peroxidase stain, which are not well resolved in Figure 2, appear to correspond to layers " $\mathrm{a}-\mathrm{c}$," and there appears to be no staining associated with layer "d." The deepest layer of immunoperoxidase stain appears to correspond in position to a combination of projection layers " $\mathrm{e}$ " and " $\mathrm{f}$," and there is no peroxidase stain associated with projection layer "g." A correspondence between the location of some of the retinal afferents within the tectum and the location of AChR-like immunoreactivity is consistent with the hypothesis that $\mathrm{AChR}$-like molecules are postsynaptic to the terminals of a subset of retinal ganglion cell types.

If the AChR-like molecules in the tectum were postsynaptic, then immunoperoxidase staining as seen in the electron microscope should be located within the synaptic cleft. In fact, virtually all peroxidase staining was found extrasynaptically. Figure 3 contains 2 electron micrographs showing that peroxidase reaction product is associated with membranes, but not at synaptic sites. Staining was typically seen on pairs of opposing membranes, a likely result of the known ability of diaminobenzidine reaction product to diffuse some distance from its site of gen-
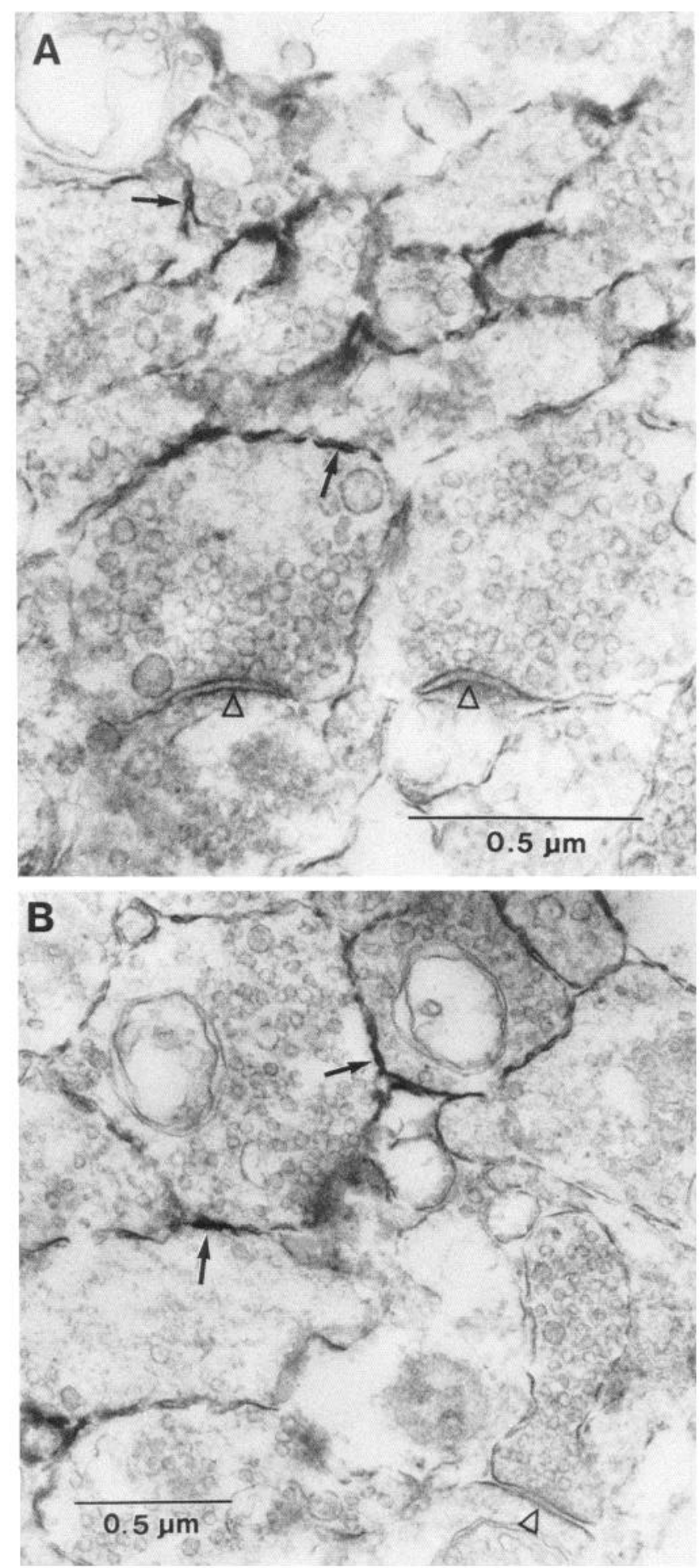

Figure 3. Electron micrographs of the optic neuropil showing extrasynaptic location of AChR-like immunoreactivity. $A$ and $B$ show 2 fields taken from the neuropil. Peroxidase stain obtained using $\mathrm{mAb} 22$ and the $\mathrm{ABC}$ technique (arrows) is associated with membranes but not with either the pre- or the postsynaptic membrane at synapses (open arrowheads).

eration and precipitate on nearby substrates (Courtoy et al., 1983). Usually, one or both of the stained membranes enclosed a cluster of synaptic vesicles, suggesting that the reaction product is associated with nerve terminals. Although we found only 1 


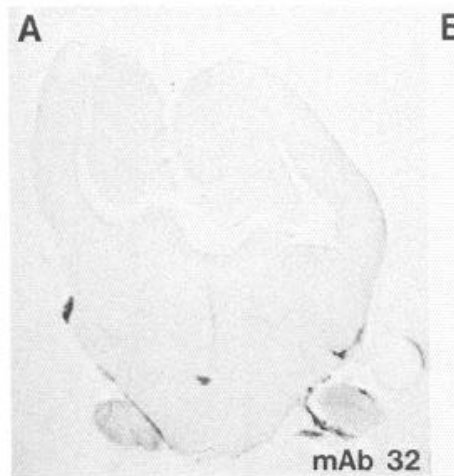

B

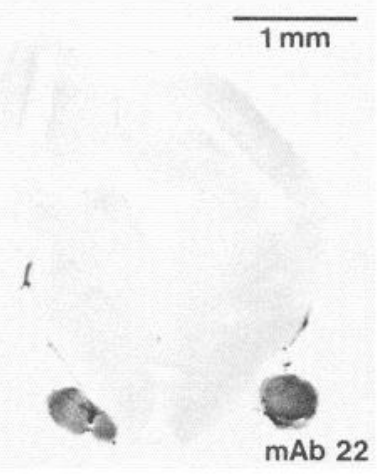

Figure 4. AChR-like immunoreactivity is associated with the optic nerves. The optic nerves in these $100 \mu \mathrm{m}$ Vibratome sections are peroxidase-stained with $\mathrm{mAb} 22(B)$ but not with control mAb $32(A)$. In these sections the optic nerves are the only structures that show specific staining (note the absence of staining in the cerebral cortex).

or 2 examples of staining in the synaptic cleft out of several hundred synapses examined, we cannot say with certainty that AChR-like molecules are absent from synaptic clefts. When tectal slices were incubated with concentrated solutions of ferritin or $6 \mathrm{~nm}$ colloidal gold, particles were found in all extracellular spaces except within synaptic clefts (data not shown). This suggests that there is a permeability barrier within synaptic clefts to macromolecules; this barrier may prevent the HRPavidin complex from entering the cleft. Thus, the electron microscopic examination of immunoreactivity indicates that there are AChR-like molecules within the frog tectum at extrasynaptic sites, but it does not rule out the possibility that such molecules are also present synaptically.

If the AChR-like molecules in the tectum were associated with retinal terminals and axons, then one might expect to find immunoperoxidase staining in the optic tract and optic nerve following incubation of tissue slices with anti-AChR mAbs. In fact, Figure $1 A$ and $2 A$ indeed show positive immunoreactivity associated with the lateral optic tract (arrows), which carries retinal axons into the tectal layer in which they will ultimately terminate. Figure 4 shows staining of the optic nerves in a coronal section through the forebrain (note the absence of staining in the cerebral cortex). mAb 22, but not mAb 32, produces dark immunoperoxidase staining in the nerves. This staining suggests that retinal axons contain AChR-like molecules, although it does not rule out the possibility that the molecules are associated with glial cells.

Another prediction of the hypothesis that AChR-like molecules in the tectum are associated with the surface of projecting retinal ganglion cell axons is that the immunoperoxidase staining seen in the tectum after incubation with anti-AChR mAbs should disappear after removal of the retina (see Scott, 1973; Ostberg and Norden, 1979). Figure $5 \mathrm{~A}$ shows an immunoperoxidase stain of a tectal slice a month after removal of the animal's left eye. The right side of the slice corresponds to the animal's right side, the side receiving the crossed retinal projection interrupted by enucleation. Although the staining is reduced a month after deafferentation, it is not abolished. Staining corresponding to layers "a," "c," and "e" remains (especially layer "c," delineated by arrows), while that associated with layers " $b$," "d," and " $f$ " is absent. While a month would ordinarily be sufficient to allow complete degeneration of afferents, Matsumoto and Scalia (1981) have shown that the retinal projec-
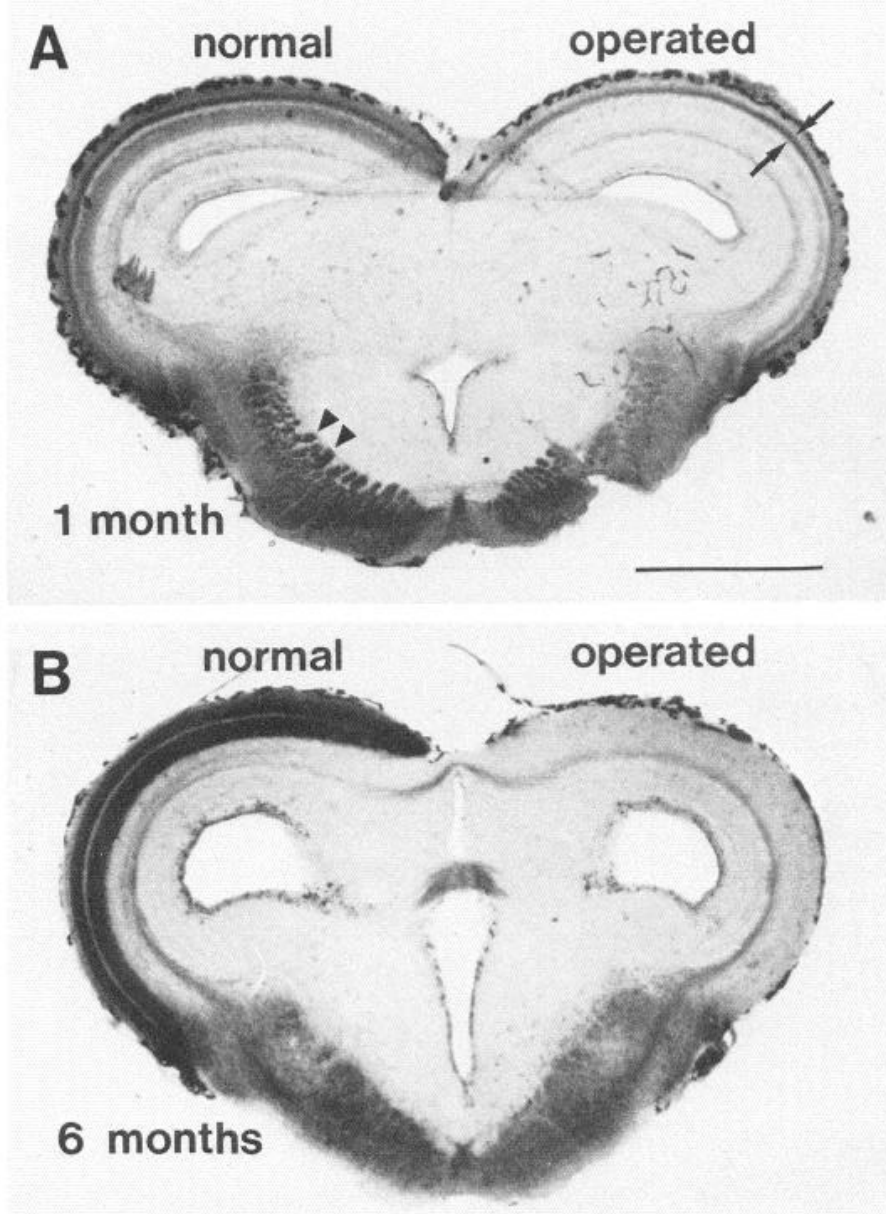

Figure 5. Loss of AChR-like immunoreactivity following removal of the retina. $A$, Removal of one retina results in partial loss of AChRlike immunoreactivity obtained with $\mathrm{mAb} 22$ in the contralateral tectum $30 \mathrm{~d}$ after surgery. The residual staining is located in bands corresponding to projection layers " $a$," " $c$," and " $e$." Staining associated with layer " $c$ " (delineated by the arrows) is most obvious, while that associated with layers " $a$ " and " $e$ " are most evident near the lateral optic tract. Matsumoto and Scalia (1981) showed that projection layers " $a$," "c," and " $e$," which are unmyelinated, survive for long periods after eye enucleation. $B$, Removal of the retina 6 months prior to staining resulted in a complete loss of immunoreactivity. Staining at the base of the tectum on both normal and operated sides (see arrowheads in $A$ ) corresponds to cobalt staining of myelinated tracts and not to HRP reaction product. Scale bar (in $A$ ): $1 \mathrm{~mm}$.

tions to layers "a," "c," and "e," which are the unmyelinated projections, degenerate only very slowly in frogs after loss of their cell bodies (see also Scott, 1973). We have confirmed their results and find that retinal axons to layers "a," "c," and "e" can survive for as long as $5 \frac{1 / 2}{2}$ months (data now shown). When survival times are increased to 5-7 months, there is complete, or nearly complete loss of immunoperoxidase staining (Fig. $5 B$; in 2 of 5 animals surviving for 5-7 months we observed residual, light peroxidase staining in layer "c"). This finding is consistent with the possibility that the AChR-like immunoreactivity is associated with retinal afferents. However, the data are also consistent with the immunoreactivity being located on the surface of tectal neurons, since the postsynaptic apparatus appears to be phagocytized after deafferentation (Ostberg and Norden, 1979). 

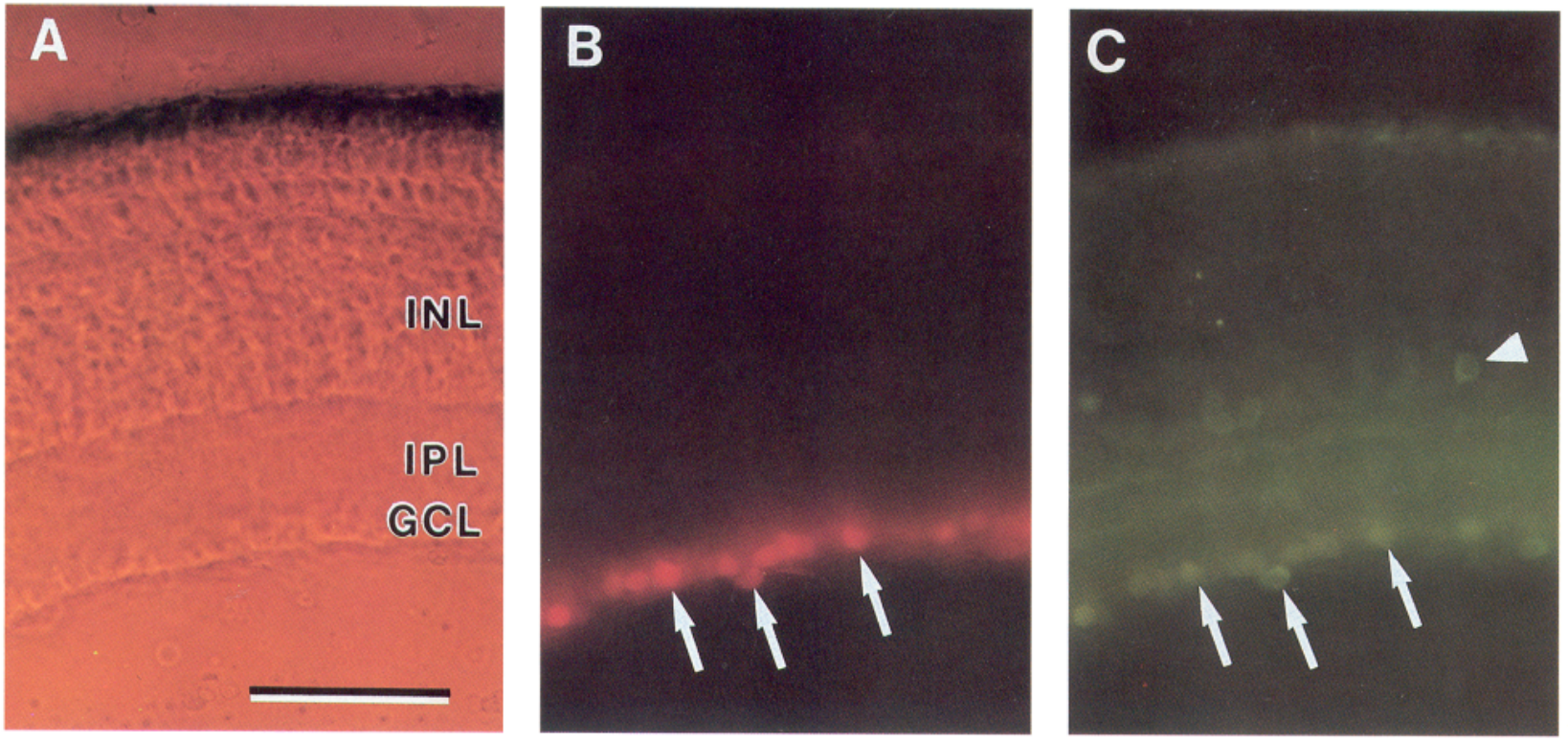

Figure 6. Retinal ganglion cells bear AChR-like molecules. A, Micrograph of a Vibratome slice of retina taken using phase optics. The following layers are labeled: ganglion cell layer $(G C L)$, inner plexiform layer $(I P L)$, and inner nuclear layer $(I N L)$. The dark tissue at the top of the retina is the pigment epithelial cell layer. $B$, Photomicrograph of the same field taken using a Zeiss filter cube with a $546 / 10 \mathrm{~nm}$ interference filter for incident light and a $590 \mathrm{~nm}$ long pass filter for emitted light, illustrating rhodamine-backfilled retinal ganglion cells within the ganglion cell layer (arrows). $C$, Photomicrograph taken using a Zeiss filter cube with a $450-490 \mathrm{~nm}$ bandpass filter for incident light and a $520 \mathrm{~nm}$ long pass filter for emitted light, illustrating fluorescein-labeled AChR-like immunoreactivity within the same slice produced by incubation of the section in mAb 22 and then in fluorescein-labeled goat anti-rat IgG. The same 3 ganglion cells are illustrated with arrows in $B$ and $C$. Many of the backfilled ganglion cells are immunoreactive. Additional staining is seen within the inner plexiform layer, where it takes on a stratified appearance. Some cell bodies near the inner border of the inner nuclear layer are also labeled (see arrowhead). Scale bar; $90 \mu \mathrm{m}$.

Immunoperoxidase staining using anti-AChR mAbs can also be demonstrated in the retina, where staining is found associated with cell bodies and their processes in both the ganglion cell layer and the inner nuclear layer and also with several diffuse bands within the inner plexiform layer (Fig. 6, right micrograph). To test the possibility that the cell bodies bearing AChR-like immunoreactivity are ganglion cells, we performed a doublelabel experiment in which retinal ganglion cells were backfilled by applying tetramethylrhodamine isothiocyanate crystals to the peripheral stump of the cut optic nerve. After 12-16 hr the retina was removed, fixed, and Vibratome-sectioned, and AChR-like immunoreactivity in retinal slices was visualized using $\mathrm{mAb} 22$ and fluorescein goat anti-rat IgG. The results show that backfilled retinal ganglion cells indeed bear AChR-like molecules (Fig. 6). Since we found no examples of ganglion cells displaced to the inner nuclear layer, the immunoreactive cells there must represent a second population of retinal neurons bearing AChRlike molecules. The diffuse, layered staining within the inner plexiform layer may represent the presence of AChR-like immunoreactivity on parts of ganglion cell dendrites, but we cannot rule out other cellular sources.

The limited number of the mAbs that cross-react with the AChR-like molecule within the tectum suggest that its similarity with skeletal muscle AChRs is limited. This conclusion was extended by our failure to demonstrate that the AChR-like molecule binds snake toxins. Neither HRP- $\alpha$-bungarotoxin nor $\alpha$-cobratoxin bound detectably to tectal slices, yet both toxins do bind to AChRs in Rana pipiens skeletal muscle and HRP$\alpha$-bungarotoxin binds to the optic neuropil in goldfish (data not shown). Furthermore, we were unable to immunoprecipitate any ${ }^{125} \mathrm{I}$ - $\alpha$-bungarotoxin binding material from extracts of Rana tec- tum using mAb 22 and goat anti-rat $\mathrm{IgG}$, yet from extracts of goldfish brain we did immunoprecipitate 5-10 fmol of bound toxin per tectum, in keeping with the results of Henley et al. (1986a). These results suggest that the tectum, at least in Rana pipiens, does not contain detectable amounts of $\alpha$-bungarotoxin binding material that cross-react with these mAbs.

\section{Discussion}

The results reported here suggest that AChR-like molecules in the frog optic tectum may be located on the surface of afferent terminals of retinal ganglion cells. This finding is consistent with other studies in fish, chicken, and rats (Henley et al., 1986a, b; Swanson et al., 1987). The results of Henley et al. (1986a, b) are particularly interesting, inasmuch as they also examined the optic tectum and used several of the same anti-electric organ AChR antibodies that were employed in this study. Henley et al. (1986b) were able to immunoprecipitate radioactivity from tectal extracts using anti-AChR mAbs several days after injecting radioactive amino acid into the eye. This demonstrates conclusively that these AChR-like molecules are synthesized in the retina and transported to the tectum along retinal axons, although it does not reveal whether the molecules resident within the tectum are pre- or postsynaptic. A similar situation appears to apply to retinal projections to subcortical structures in rats (Swanson et al., 1987). Here, AChR-like immunoreactivity is found in the superior colliculus and in the pathway from the retina to the colliculus. As also found in the present study, the immunoreactivity in rat colliculus disappears following removal of the eye (Swanson et al., 1987; see also Swanson et al., 1983; Clarke et al., 1986). In the present paper, we report the first electron microscopic immunocytochemical study of AChR-like 
immunoreactivity in the CNS. A novel finding of this work is the demonstration that the tectum contains AChR-like moleculcs which are extrasynaptic.

The discovery of AChR-like molecules in the frog retina is interesting in light of numerous studies in several species indicating that some amacrine cells are cholinergic (Masland et al., 1984; Pourcho and Osman, 1986; Tumosa and Stell, 1986; Voigt, 1986; Millar and Morgan, 1987; see also Baughman and Bader, 1977) and that ganglion cells in rats bear functional AChRs (Lipton et al., 1987). The immunoperoxidase staining of at least some of the cell bodies within the ganglion cell layer is attributable to staining of ganglion cells themselves, as demonstrated by simultaneously backfilling these cells from the optic nerve. The cell bodies bearing AChR-like immunoreactivity in the inner nuclear layer may be amacrine cells based upon their proximity to the inner plexiform layer. Both ganglion cells and amacrine cells in chick retina also bear AChR-like immunoreactivity (Keyser et al., 1988). The laminar staining within the inner plexiform layer (Fig. 6C) is reminiscent of the laminar distribution of choline acetyltransferase in the inner plexiform layer of other species (Tumosa et al., 1984; Pourcho and Osman, 1986; Voigt, 1986; Famiglietti and Tumosa, 1987; Spira et al., 1987) and is likely to represent regionally specific AChR-like molecules on the processes of AChR-bearing cells, possibly ganglion cells. It is interesting to note that retinas in several species have a nonuniform, laminar distribution of synapses in the inner plexiform layer (Dubin, 1970; Koontz and Hendrickson, 1987). The selective labeling of a subset of these synapses might well be expected to produce a laminar pattern of immunocytochemical staining of the sort observed (Fig. 6C).

The antigenic structure of the AChR-like molecule within the frog tectum can be inferred from examining the specificity of the $\mathrm{mAbs}$ that recognize it. It is clear that this molecule possesses an MIR very similar to that on true AChRs in frog skeletal muscle, since 22 anti-MIR $\mathrm{m} \Lambda$ bs recognized both molcculcs. The MIR is a highly conserved region known to be located on the extracellular surface of the $\alpha$ subunit. The MIR has recently been shown to consist, at least in part, of sequence $\alpha 67-76$ in human muscle (Tzartos et al., 1988), $\alpha 61-76$ in mouse muscle (Barkas et al., 1988), and $\alpha 63-83$ in Torpedo electric organ AChR (M. Das and J. Lindstrom, unpublished observations). The frog neuronal AChR-like molecule is likely to display sequence homology with human and Torpedo AChR in this region. Homology elsewhere may be limited, however, since only 6 of 16 non-anti-MIR mAbs that bound AChRs in muscle cross-reacted in the tectum, and in each instance cross-reactivity was weak and detected in only some of the experiments (Table 1). All 16 non-MIR mAbs tested are known to recognize intracellular epitopes (Sargent et al., 1984a). The "poor showing" of these mAbs is not the result of their inability to penetrate AChR-bearing profiles, since saponin renders these profiles permeable to large macromolecules. Rather, it is likely that there is less homology between the cytoplasmic portions of the AChR from brain and from muscle/electric organ. Comparison of cDNA sequences reveals that a long sequence corresponding to the immunogenic cytoplasmic surface of AChRs from electric organ is poorly conserved and is unique between corresponding muscle AChR subunits of different species and corresponding AChR subunits of muscle and nerve (reviewed in Lindstrom et al., 1987).

The failure to demonstrate that $\alpha$-bungarotoxin or cobratoxin bind to the frog tectum is consistent with other data suggesting that in several neural tissues $\alpha$-bungarotoxin does not recognize neuronal AChRs (Patrick and Stallcup, 1977; Carbonetto et al., 1978; Ravdin and Berg, 1979; Jacob and Berg, 1983; Smith et al., 1983; Loring et al., 1984; Clarke et al., 1985; Swanson et al., 1987). In a few vertebrate tissues, however, $\alpha$-bungarotoxin does bind to AChRs, or at least to molecules that bear homology with AChRs, as determined by antibody cross-reaction. One such instance is the goldfish optic tectum (Henley et al., 1986a), and another is bullfrog sympathetic ganglia (Marshall, 1981). $\mathrm{AChRs}$ and $\alpha$-bungarotoxin-binding proteins are one and the same molecule in electric organ and muscle but separate molecules in both the CNS and peripheral ganglia of many vertebrates. It is possible that one will not be able to generalize about the identity of $\alpha$-bungarotoxin-binding proteins in ganglia and CNS tissue since in some instances $\alpha$-bungarotoxin binds AChR and in others it does not.

In combination with the work of Henley et al. (1986a, b), these results indicate that nicotinic AChR-like molecules in the optic tectum are synthesized in the retina, are transported to the tectum, and may be located on the surfaces of retinal afferents there. However, neither the metabolic labeling experiments of Henley et al. (1986a, b) nor our own work demonstrates conclusively that $\mathrm{AChR}$-like molecules within the tectum are associated with retinal terminals. It is conceivable that they are transported transsynaptically and then reside on the surface of tectal neurons. This possibility seems remote, however, since it implies that neurons may ordinarily synthesize receptors and transport them to their terminals only to pass them on to other cells. In addition, the high incidence of immunoperoxidase staining at cell surfaces where 2 vesicle-bound profiles are apposed (Fig. 3) indicates that immunoreactivity is present on the plasma membrane of nerve terminals. Given the association of AChR-like molecules with more proximal parts of the retinal ganglion cell axon, it is only natural to suppose that they are present on its terminal as well.

Our electron microscopic evidence indicates, surprisingly, that the tectum contains AChR-like molecules that are extrasynaptic. It is natural to wonder what possible function could be served by extrasynaptic $\mathrm{AChRs}$ and to wonder whether these molecules are AChRs at all. Although it would seem unlikely, we cannot rule out the possibility that these molecules serve a functional role only in the retina and are transported "mistakenly" by retinal ganglion cells to the tectum. Another possibility is that these molecules resemble AChRs antigenically but do not serve a functional role as ligand-gated ion channels. [However, Lipton et al. (1987) have demonstrated the existence of functional AChRs on retinal ganglion cells in rat.] A more intriguing possibility is that AChR-like molecules do act as receptors within the tectum, albeit in a nonclassical way. The principal cholinergic input to the optic tectum is from the nucleus isthmi (Ricciuti and Gruberg, 1985; Ross and Godfrey, 1986; Desan et al., 1987), and it is possible that ACh released from tectal terminals of neurons originating in the nucleus isthmi might diffuse some distance before acting on AChRs, be they on the surface of retinal terminals and/or tectal neurons. The function of this paracrine system might be to modulate the release properties of the ganglion cell terminals and/or the responsiveness of their targets. One possible difficulty with this notion is that choline acetyltransferase (arising from terminals of nucleus isthmi neurons) and AChR-like molecules are not completely coextensive: The transferase is missing from projection layer " $b$ " (Desan ct al., 1987), and the AChR-like molecules are missing from projection layers of "d" and "g." Nevertheless, it would be worth- 
while examining the fine structure of nucleus isthmi-derived terminals within the tectum to learn if any of them have the "distributed" characteristics of terminals that do not release transmitter focally onto individual target cells (e.g., Descarries et al., 1975).

The original studies in goldfish on $\alpha$-bungarotoxin and nicotinic agonists and antagonists suggested that retinotectal connections might be nicotinic in goldfish and toad (Freeman et al., 1980; Oswald et al., 1980; Oswald and Freeman, 1981). A number of more recent studies have cast doubt on this scheme. Retinal ganglion cells in a variety of species do not appear to be cholinergic (e.g., Tumosa and Stell, 1986), and the choline acetyltransferase within the tectum can be accounted for by projections to the tectum from the nucleus isthmi alone (Ricciuti and Gruberg, 1985; Ross and Godfrey, 1986; Desan et al., 1987). Finally, recent pharmacological studies on the goldfish system suggest that nicotinic antagonists do not block the direct response of tectal neurons to excitation of retinal ganglion cell axons (Langdon and Freeman, 1987). In fact, the most likely candidate transmitter for the direct retinotectal input is an amino acid (Langdon and Freeman, 1987). Cholinergic agonists and antagonists do have intriguing effects on retinotectal transmission (Freeman, 1977; Schmidt, 1985; Langdon and Freeman, 1987). The mechanism by which these effects are exerted is likely to lead to new insights into neural processing.

\section{References}

Barkas, T., J.-M. Gabriel, A. Mauron, G. J. Hughes, B. Roth, C. Alliod, S. J. Tzartos, and M. Balliret (1988) Monoclonal antibodies to the main immunogenic region of the nicotinic acetylcholine receptor bind to residues 61-76 of the $\alpha$ subunit. J. Biol. Chem. 263: 5916-5920.

Baughman, R. W., and C. R. Bader (1977) Biochemical characterization and cellular localization of the cholinergic system in the chicken retina. Brain Res. 138: 469-485.

Carbonetto, S. T., D. M. Fambrough, and K. J. Muller (1978) Nonequivalence of $\alpha$-bungarotoxin receptors and acetylcholine receptors in chick sympathetic neurons. Proc. Natl. Acad. Sci. USA 75: 10161020 .

Clarke, P. B. S., R. D. Schwartz, S. M. Paul, C. B. Pert, and A. Pert (1985) Nicotinic binding in rat brain: Autoradiographic comparison of $\left[{ }^{3} \mathrm{H}\right]$ acetylcholine, $\left[{ }^{3} \mathrm{H}\right]$ nicotine, and $\left[{ }^{125} \mathrm{I}\right]-\alpha$-bungarotoxin. J. Neurosci. 5: 1307-1315.

Clarke, P. B. S., G. S., Hamill, N. S. Nadi, D. Jacobowitz, and A. Pert (1986) ${ }^{3} \mathrm{H}$-nicotine- and ${ }^{125} \mathrm{I}-\alpha$-bungarotoxin-labeled nicotinic receptors in the interpeduncular nucleus of rats. II. Effects of habenular deafferentation. J. Comp. Neurol. 251: 407-413.

Courtoy, P. J., D. H. Dicton, and M. G. Farquhar (1983) Resolution and limitation of the immunoperoxidase procedure in the localization of extracellular matrix antigens. J. Histochem. Cytochem. 31: 945951.

Desan, P. H., E. R. Gruberg, K. M. Grewell, and F. Eckenstein (1987) Cholinergic innervation of the optic tectum in the frog Rana pipiens. Brain Res. 413: 344-349.

Descarries, L., A. Beaudet, and K. C. Watkins (1975) Serotonin nerve terminals in adult rat neocortex. Brain Res. 100: 563-588.

Dubin, M. W. (1970) The inner plexiform layer of the vertebrate retina: A quantitative and comparative electron microscopic analysis. J Comp Neurol. 140: 479-506.

Famiglietti, E. V., and N. Tumosa (1987) Immunocytochemical staining of cholinergic amacrine cells in rabbit retina. Brain Res. 413:398403.

Freeman, J. A. (1977) Possible regulatory function of acetylcholine receptor in maintenance of retinotectal synapses. Nature 269: $218-$ 222.

Freeman, J. A., J. T. Schmidt, and R. E. Oswald (1980) Effect of $\alpha$-bungarotoxin on retinotectal transmission in the goldtish and toad. Neuroscience 5: 929-942.

Henley, J. M., M. Mynlieff, J. M. Lindstrom, and R. E. Oswald (1986a)
Interaction of monoclonal antibodies to electroplaque acetylcholine receptors with the $\alpha$-bungarotoxin binding site of goldfish brain. Brain Res. 364: 405-408

Henley, J. M., J. M. Lindstrom, and R. E. Oswald (1986b) Acetylcholine receptor synthesis in retina and transport to optic tectum in goldfish. Science 232: 1627-1629.

Hsu, S.-M., L. Raine, and H. Fanger (1981) Use of avidin-biotinperoxidase complex $(\mathrm{ABC})$ in immunoperoxidase techniques: A comparison between $\mathrm{ABC}$ and unlabeled antibody (PAP) procedures. $J$. Histochem. Cytochem. 29: 577-580.

Itoh, K., A. Konishi, S. Nomura, N. Mizuno, Y. Nakamura, and T. Sugimoto (1979) Application of coupled oxidation reaction to clectron microscopic demonstration of horseradish peroxidase: Cobaltglucose oxidase method. Brain Res. 175: 341-346.

Jacob, M. H., and D. K. Berg (1983) The ultrastructural localization of $\alpha$-bungarotoxin binding sites in relation to synapses on chick ciliary ganglion neurons. J. Neurosci. 3: 260-271.

Keyser, K. T., T. E. Hughes, P. J. Whiting, J. M. Lindstrom, and H. J. Karten (1988) Cholinoceptive neurons in the retina of the chick: An immunohistochemical study of the nicotinic acetylcholine receptors. Visual Neurosci. 1: 349-366.

King, J. C., R. M. Lechan, G. Kugel, and E. L. P. Anthony (1983) Acrolein: A fixative for immunocytochemical localization of peptides in the central nervous system. J. Histochem. Cytochem. 31: 62-68.

Koontz, M. A., and A. E. Hendrickson (1987) Stratified distribution of synapses in the inner plexiform layer of primate retina. J. Comp. Neurol. 263: 581-592.

Langdon, R. B., and J. A. Freeman (1987) Pharmacology of retinotectal transmission in the goldfish: Effects of nicotinic ligands, strychnine, and kynurenic acid. J. Neurosci. 7: 760-773.

Lindstrom, J., B. Einarson, and S. Tzartos (1981) Production and assay of antibodies to acetylcholine receptors. Methods Enzymol. 74: $432-460$.

Lindstrom, J., R. Schoepfer, and P. Whiting (1987) Molecular studies of the neuronal nicotinic acetylcholine receptor family. Mol. Neurobiol. 1: 281-337.

Lipton, S. A., E. Aizeman, and R. H. Loring (1987) Neural nicotinic acetylcholine responses in solitary mammalian retinal ganglion cells. Pfluegers Arch. 410: 37-43.

Loring, R. H., V. A. Chiappinnelli, R. E. Zigmond, and J. B. Cohen (1984) Characterization of a snake venom neurotoxin which blocks nicotinic transmission in the avian ciliary ganglion. Neuroscience 11 : 989-999.

Marshall, L. M. (1981) Synaptic localization of $\alpha$-bungarotoxin binding which blocks nicotinic transmission at frog sympathetic neurons. Proc. Natl. Acad. Sci. USA 78: 1948-1952.

Masland, R. H., J. W. Mills, and S. A. Hayden (1984) Acetylcholinesynthesizing amacrine cells: Identification and selective staining by using radioautography and fluorescent markers. Proc. R. Soc. London [Biol.] 223: 79-100.

Matsumoto, D. E., and F. Scalia (1981) Long-term survival of centrally projecting axons in the optic nerve of the frog following destruction of the retina. J. Comp. Neurol. 202: 135-155.

Maturana, H. R., J. Y. Lettvin, W. S. McCulloch, and W. H. Pitts (1960) Anatomy and physiology of vision in the frog (Rana pipiens). J. Gen. Physiol. 43: 129-175.

McLean, I. W., and P. K. Nakane (1974) Periodate-lysine-paraformaldehyde fixative: A new fixative for immunoelectron microscopy. J. Histochem. Cytochem. 22: 1077-1083.

Millar, T. J., and I. G. Morgan (1987) Cholinergic amacrine cells in the rabbit retina synapse onto other cholinergic amacrine cells. Neurosci. Lett. 74: 281-285.

Mühlpfordt, H. (1982) The preparation of colloidal gold particles using tannic acid as an additional reducing agent. Experientia 38: 11271128.

Ostberg, A., and J. Norden (1979) Ultrastructural study of degeneration and regeneration in the amphibian tectum. Brain Res. 168:441455.

Oswald, R. E., and J. A. Freeman (1981) $\alpha$-Bungarotoxin binding and central nervous system nicotinic acetylcholine receptors. Neuroscience $6: 1-14$.

Oswald, R. E., J. T. Schmidt, J. J. Norden, and J. A. Freeman (1980) Localization of $\alpha$-bungarotoxin binding sites to the goldfish retinotectal projection. Brain Res. 187: 113-127.

Patrick, J., and W. B. Stallcup (1977) Immunological distinction between acetylcholine receptor and the $\alpha$-bungarotoxin-binding com- 
ponent on sympathetic neurons. Proc. Natl. Acad. Sci. USA 74:46894692.

Potter, H. D. (1969) Structural characteristics of cell and fiber populations in the optic tectum of the frog (Rana catesbiana). J. Comp. Neurol. 136: 203-232.

Pourcho R. G., and K. Osman (1986) Cytochemical localization of cholinergic amacrine cells in cat retina. J. Comp. Neurol. 247: 497504.

Ravdin, P. M., and D. K. Berg (1979) Inhibition of neuronal acetylcholine sensitivity by $\alpha$-toxins from Bungarus multicinctus venom. Proc. Natl. Acad. Sci. USA 76: 2072-2076.

Ricciuti, A. J., and E. R. Gruberg (1985) Nucleus isthmi provides most tectal choline acetyltransferase in the frog Rana pipiens. Brain Res. 341: 399-402.

Ross, C. D., and D. A. Godfrey (1986) Effect of enucleation on choline acetyltransferase activity in layers of goldfish optic tectum. Brain Res. 373: 49-56.

Sargent, P. B., B. E. Hedges, L. Tsavaler, L. Clemmons, S. Tzartos, and J. M. Lindstrom (1984a) The structure and transmembrane nature of the nicotinic acetylcholine receptor in amphibian muscle as re vealed by cross-reacting monoclonal antibodies. J. Cell Biol. 98: 609618.

Sargent, P. B., S. H. Pike, L. Tsavaler, and J. M. Lindstrom (1984b) Monoclonal antibodies to electric organ acetylcholine receptors bind to frog optic tectum. Soc. Neurosci. Abstr. 10:935.

Schmidt, J. T. (1985) Apparent movement of optic terminals out of a local postsynaptically blocked region in goldfish optic tectum. $\mathbf{J}$ Neurophysiol. 53: 237-251.

Scott, T. M. (1973) Degeneration of optic nerve terminals in the frog tectum. J. Anat. 114: 261-269.

Slot, I. W., and H. J. Geuze (1981) Sizing of protein A-colloidal gold probes for immunoelectron microscopy. J. Cell Biol. 90: 533-536.

Smith, M. A., J. F. Margiotta, and D. K. Berg (1983) Differential regulation of acetylcholine sensitivity and $\alpha$-bungarotoxin-binding sites on ciliary ganglion neurons in cell culture. J. Neurosci. 3: 23952402.

Spira, A. W., T. J. Millar, I. Ishimoto, M. L. Epstein, C. D. Johnson,
J. L. Dahl, and I. G. Morgan (1987) Localization of choline acetyltransferase-like immunoreactivity in the embryonic chick retina. J. Comp. Neurol. 260: 526-538.

Swanson, L. W., J. Lindstrom, S. Tzartos, L. C. Schmued, D. D. M. O'Leary, and W. M. Cowan (1983) Immunohistochemical localization of monoclonal antibodies to the nicotinic acetylcholine receptor in chick midbrain. Proc. Natl. Acad. Sci. USA 80:4532-4536.

Swanson, L. W., D. M. Simmons, P. J. Whiting, and J. Lindstrom (1987) Innmunohistochemical localization of neuronal nicotinic receptors in the rodent central nervous system. J. Neurosci. 7:3334 3342.

Tumosa, N., and W. K. Stell (1986) Choline acetyltransterase immunoreactivity suggests that ganglion cells in the goldfish retina are not cholinergic. J. Comp.Neurol. 244: 267-275.

Tumosa, N., F. Eckenstein, and W. K. Stell (1984) Immunocytochemical localization of putative cholinergic neurons in the goldfish retina. Neurosci. Lett 48:255-259.

Tzartos, S. J., and J. M. Lindstrom (1980) Monoclonal antibodies used to probe acetylcholine receptor structure: Localization of the main immunogenic region and detection of similaritics between subunits. Proc. Natl. Acad. Sci. USA 77: 755-759.

Tzartos, S. J., D. E. Rand, B. L. Einarson, and J. M. Lindstrom (1981) Mapping of surface structures of Electrophorus acetylcholine receptor using monoclonal antibodies. J. Biol. Chem. 256: 8635-8645.

Tzartos, S., L. Langeberg, S. Hochschwender. L. W. Swanson, and J. Lindstrom (1986) Characteristics of monoclonal antibodies to denatured Torpedo and to native calf acetylcholine receptors: Species, subunit and region specificity. J. Neuroimmunol. 10: 235-253.

Tzartos, S. J., A. Kokla, S. L. Walgrave, and B. M. Conti-Tronconi (1988) Localization of the main immunogenic region of human muscle acetylcholine to residues 67-76 of the $\alpha$ subunit. Proc. Natl. Acad. Sci. USA 85: 2899-2903.

Voigt, T. (1986) Cholinergic amacrine cells in the retina. J. Comp. Neurol. 248: 1935

Wray, B. E., and R. Sealock (1984) Ultrastructural immunocytochemistry of particulate fractions using polyvinyl chloride microculture wells. J. Histuchem. Cylochem. 31: 1117-1120. 